$\begin{array}{cl}\text { PRAMANA } & \text { (C) Indian Academy of Sciences } \\ \begin{array}{c}\text { journal of } \\ \text { physics }\end{array} & \begin{array}{l}\text { Vol. } 65, \text { No. } 4 \\ \text { October } 2005 \\ \text { pp. } 571-579\end{array}\end{array}$

\title{
Flow-driven voltage generation in carbon nanotubes
}

\author{
A K SOOD ${ }^{1,2}, \mathrm{~S} \mathrm{GHOSH}^{1}$ and ANINDYA DAS ${ }^{1}$ \\ ${ }^{1}$ Department of Physics, Indian Institute of Science, Bangalore 560 012, India \\ ${ }^{2}$ DST Unit of Nanoscience and Nanotechnology, Indian Institute of Science, \\ Bangalore 560 012, India and Jawaharlal Nehru Centre for Advanced Scientific Research, \\ Jakkur P.O., Bangalore 560 064, India \\ E-mail: asood@physics.iisc.ernet.in
}

\begin{abstract}
The flow of various liquids and gases over single-walled carbon nanotube bundles induces an electrical signal (voltage/current) in the sample along the direction of the flow. The electrical response generated by the flow of liquids is found to be logarithmic in the flow speed over a wide range. In contrast, voltage generated by the flow of gas is quadratically dependent on the gas flow velocity. It was found that the underlying physics for the generation of electrical signals by liquids and gases are different. For the liquid, the Coulombic interaction between the ions in the liquid and the charge carriers in the nanotube plays a key role while electrical signal generation due to gas flow is due to an interplay of Bernoulli's principle and Seebeck effect. Unlike the liquid case which is specific to the nanotubes, the gas flow effect can be seen for a variety of solids ranging from single and multi-walled carbon nanotubes, graphite and doped semiconductors.
\end{abstract}

Keywords. Nanotubes; flow sensors.

PACS Nos 78.67.Bf; 05.60.-k

\section{Introduction}

The nanotubes with their rolled-up graphene surfaces provide large surface area in atomic contact with the flowing electrolyte across the solid-liquid interface. The one-dimensionality of the conducting nanotube further facilitates charge-carrier entrainment. Thus any momentum transfer (direct or mediated) will cause the charge carriers in the nanotubes to scatter in a preferential direction along the axis, resulting in a finite electrical current. It was observed that the flow of liquid over a mat of single-walled carbon nanotube (SWNT) bundles generates electrical signals $[1,2]$. The observed voltage fits well to an empirical logarithm function of the liquid flow velocity $\left(u_{\mathrm{L}}\right)$. The magnitude of the flow-induced signals depends significantly on the ionic strength of the flowing liquid. This suggests that the mechanism involving direct driving of the charge carriers in the nanotubes by fluctuating Coulombic fields dominates over the other mechanism involving mediated transfer of momentum to the nanotube charge carriers, say via phonons. In this paper, we present 
(a)

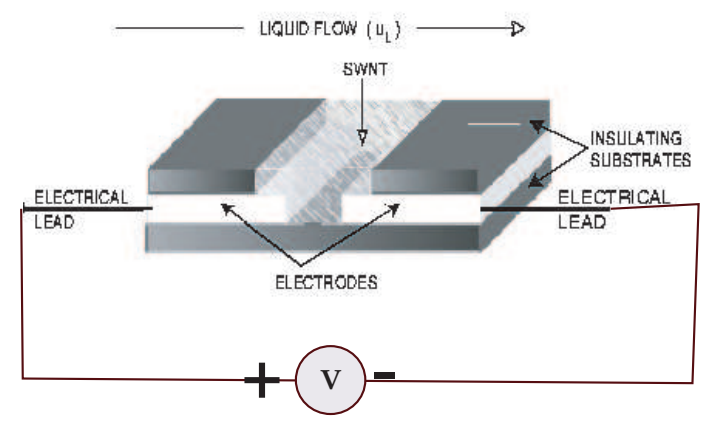

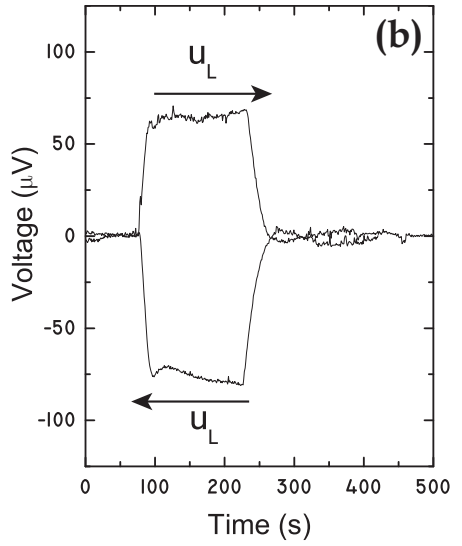

Figure 1. (a) Schematic sketch of the nanotube flow sensor placed along the flow direction $\left(u_{\mathrm{L}}\right)$. SWNT bundles are packed between two metal electrodes. The insulating substrate keeps the SWNT in place. The electrical leads are taken out from the metal electrodes. (b) A typical measurement of open-circuit voltage for the flow speed of $4 \times 10^{-4} \mathrm{~m} / \mathrm{s}$. The positive signal is for liquid flowing from left to right of the sensor, while the negative signal is for the liquid flowing from right to left.

new data related to (i) the dependence of electrical signal on the ionic concentration and length of the sample and (ii) application of our nanotube sensor to the study of unsteady flows.

Following our experiments on liquid flow, it was only natural to ask the question: Will a similar effect occur in carbon nanotubes due to the flow of gases as well? We found that the gas flow over nanotubes also generates electrical signals. However, this effect is not unique to nanotubes and occurred for a wide variety of solids, including single and multi-walled carbon nanotubes, doped semiconductors and metals [3]. The range of gas velocities covered in the experiments was 1-140 $\mathrm{m} / \mathrm{s}$. The voltage and current depend quadratically on the flow velocity; and the magnitude and sign of the voltage depend on the properties of the solid. These results will be briefly reviewed.

\section{Generation of electrical signals by flow of liquid over nanotubes}

\subsection{Experimental details, results and some applications of the flow sensor}

The SWNT bundles were prepared in Prof. Rao's laboratory by the electric-arc method followed by purification processes [4]. The randomly oriented nanotube bundles, with an average tube diameter of $1.5 \mathrm{~nm}$, were densely packed in a groove between two metal electrodes. The construction of the sensor is schematically shown in figure 1a (this has been discussed in detail in Ghosh et al. $[1,2]$ ). When the sample was immersed in water at rest, a voltage developed along the sample due to an electrochemical potential difference at the interface of the SWNT bundles with 

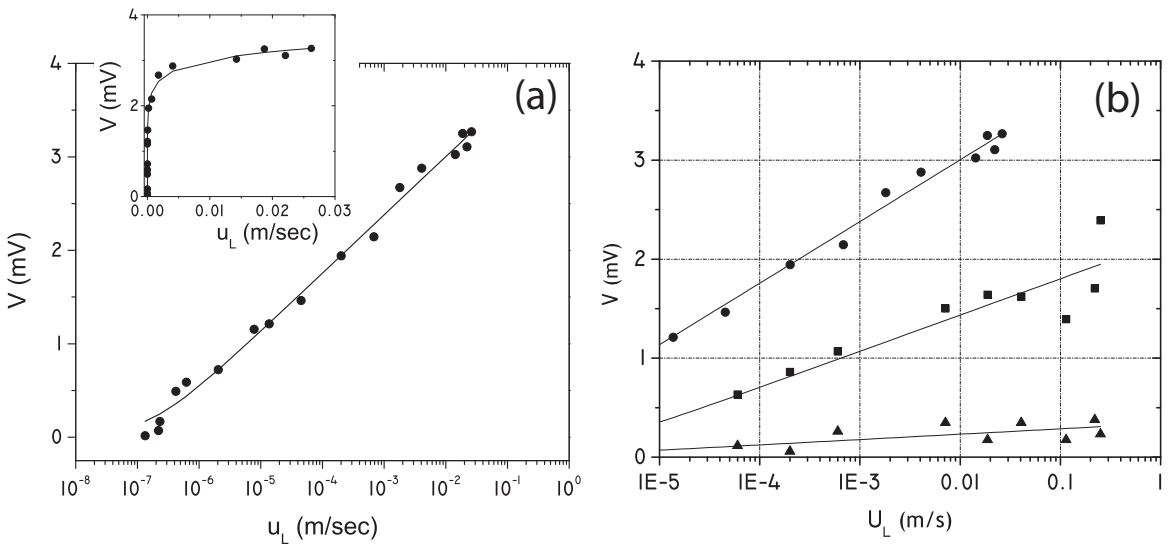

Figure 2. (a) A log-linear plot of the variation of open-circuit voltage $(V)$ developed as function of velocity $\left(u_{\mathrm{L}}\right)$ of water. Solid line is a fit to functional form $V=\alpha_{\mathrm{V}} \log \left(u_{\mathrm{L}} \beta_{\mathrm{V}}+1\right)$, with $\alpha_{\mathrm{V}}$ and $\beta_{\mathrm{V}}$ as constants. The inset shows the same data on a linear plot. (b) Variation of signal with various length's of the sample; $\ell=1 \mathrm{~mm}$ (filled circles), $\ell=0.5 \mathrm{~mm}$ (filled squares), $\ell=0.25$ $\mathrm{mm}$ (filled up-triangles). The solid lines show fit to eq. (1)

the metal electrodes. This offset voltage was subtracted from the voltage measured at finite velocities of the liquid to get the flow-induced voltage. The flow-induced voltage developed only along the flow direction, with no voltage generated in the direction perpendicular to the flow. Figure $1 \mathrm{~b}$ shows a typical measurement of voltage for the flow speed of $4 \times 10^{-4} \mathrm{~m} / \mathrm{s}$. The positive signal is for the liquid flowing from left to right of the sensor while the negative signal is for the liquid flowing from right to left of the sensor. This figure clearly shows that when the direction of the flow is reversed, the polarity of the signal across the nanotubes also changes its sign. The reversal of the flow-induced electrical signals with the change in the direction of the flow is a key feature of the effect. We have seen in the course of our experiments that if the nanotubes are not properly packed between the two electrodes, the signals generated on reversing the flow direction can be highly asymmetric, i.e., the electrical signals generated by flowing liquid in the two flow directions will be unequal. This aspect, we believe is due to the poor quality control of the electrical contacts between nanotube bundles and metal electrodes in our present way of making the sensors and needs to be addressed in future studies. It was further observed that for a given flow velocity, the magnitude of flow-induced signals for different sensors varied from few microvolts to hundreds of microvolts $[1,2]$. We attribute this large variation in signals to the different degrees of alignment of the SWNT mat with respect to the liquid flow direction and the varying specific surface area of the different SWNT samples in contact with the flowing liquid.

Figure 2a shows the observed dependence of the flow-induced open circuit voltage on the flow velocity of water [1]. The solid line in figure $2 \mathrm{a}$ is a fit to the empirical relation 


\section{A K Sood, S Ghosh and Anindya Das}

$$
V=\alpha_{\mathrm{V}} \log \left(u_{\mathrm{L}} \beta_{\mathrm{V}}+1\right),
$$

where $\alpha_{\mathrm{V}}$ and $\beta_{\mathrm{V}}$ are parameters, $\alpha_{\mathrm{V}}=0.6 \mathrm{mV}$ and $\beta_{\mathrm{V}}=6.5 \times 10^{6} \mathrm{~s} / \mathrm{m}$ for the best fit [5]. The open circuit voltage and short circuit current generated by the SWNT bundles by flowing water over them fit the empirical logarithmic law over a large range of velocity. The ratio of the voltage and current was related by the nanotube sample resistance [2]. Figure $2 \mathrm{~b}$ shows that the flow-induced voltages were found to scale with the length $(\ell)$ of the sensor parallel to the direction of the flow.

We now discuss the direction of the flow-induced current with respect to the flow direction. The voltmeter is connected across the SWNT sample as shown in figure 1a which will give a negative signal if the induced current $I$ is parallel to $u_{\mathrm{L}}$ and positive signal for $I$ anti-parallel to $u_{\mathrm{L}}$. The magnitude and the direction of the current is seen to be extremely sensitive to the nature of the electrolyte. This is shown in figure 3a for different additives like $\mathrm{CsOH}, \mathrm{LiClO}_{4}, \mathrm{KOH}$ and $\mathrm{HCl}$ to water, characterized by the ionic conductivities $\left(G_{0}\right)$ of the resulting electrolyte. The dependence of the flow-induced signal (fixed flow speed of $10^{-3} \mathrm{~m} / \mathrm{s}$ ) on the concentration of different types of ions in the liquid is highly non-trivial and nonmonotonic. Though we do not have a quantitative understanding of these results, the differences for various additives arise probably from the adsorption properties of the nanotube surfaces to different types of ions which result in different charge transfer scenarios. Further, we were able to deterministically control the direction of the flow-induced current by applying an electrochemical bias voltage $\left(V_{\mathrm{B}}\right)$ to the nanotubes with respect to a counter electrode [2] the results of which are shown in figure $3 \mathrm{~b}$ for $0.01 \mathrm{M} \mathrm{KCl}$ and $0.01 \mathrm{M} \mathrm{HCl}$. It is seen that the flow-induced signal is positive, i.e., $I$ is anti-parallel to $u_{\mathrm{L}}$ when $V_{\mathrm{B}}$ is positive, and the sign of the signal is negative, i.e., $I$ is parallel to $u_{\mathrm{L}}$, when $V_{\mathrm{B}}$ is negative, consistent with our theory $[2,6]$.

We have been able to measure the unsteady flow created at an expansion. The schematic of the experimental set-up is shown in the left panel of figure 4 . The diameter of the inlet tube was $0.6 \mathrm{~cm}$ which opens into a tube of diameter $2.5 \mathrm{~cm}$. It is known that under such conditions, that there will be eddies in the flow chamber in the expansion zone [7]. The flow in the present case is further complicated by the reflections from the top surface of the flow chamber. From dimensional analysis the frequency $(\tilde{\nu})$ of the oscillation seen in figure 4 is expected to be $\tilde{\nu}=$ $u_{\mathrm{L}} / 2 L$, where $L(=5 \mathrm{~cm})$ is the length of the flow chamber. For $u_{\mathrm{L}}=0.4 \mathrm{~cm} / \mathrm{s}$, $u_{\mathrm{L}} / 2 L=0.04 \mathrm{~Hz}$ which is close to the observed frequency of $0.05 \mathrm{~Hz}$ (see figure 4). From this experiment it was found that the response time of the sensor is better than few milliseconds. However, it needs to be stressed that the response time reported here is limited by the liquid flow velocity and the rate of data acquisition. Based on the understanding that the origin of the flow-induced electrical signals is electronic in origin we expect the response time of the sensor to be much smaller than milliseconds.

We have used the liquid flow-induced signals in developing an application of the SWNT as a vibration sensor in liquid environment [8]. The frequency range that has been tested is from $100 \mu \mathrm{Hz}$ to $1 \mathrm{kHz}$. We have also been able to construct a device based on nanotube flow sensing element to measure solid vibrations [8]. The frequency range that has been tested by us is from $0.5 \mathrm{~Hz}$ to $1 \mathrm{kHz}$. The minimum acceleration of the vibrating solid block is $\sim 10^{-3} \mathrm{~g}$, where $1 \mathrm{~g}=10 \mathrm{~m} / \mathrm{s}^{2}$. 

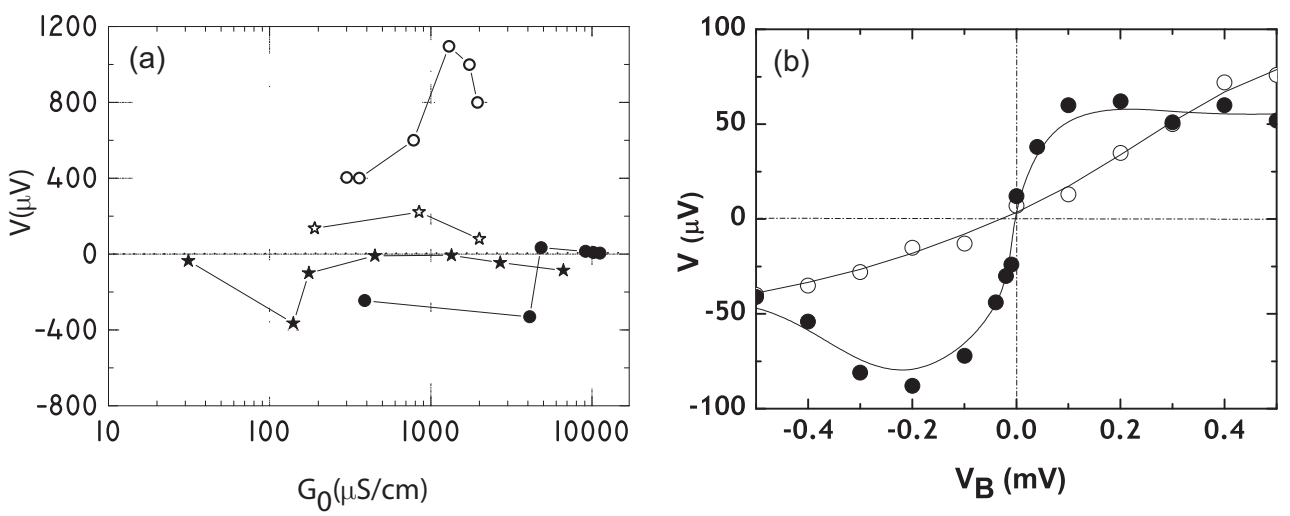

Figure 3. (a) Variation of the magnitude and the direction of the flowinduced voltages for additives of different types: $\mathrm{CsOH}$ (filled star), $\mathrm{LiClO}_{4}$ (open circle), $\mathrm{KOH}$ (filled circle), $\mathrm{HCl}$ (open star) and strengths, characterized by their conductivities $\left(G_{0}\right)$ for a fixed flow velocity, $u_{\mathrm{L}}=10^{-3} \mathrm{~m} / \mathrm{s}$. (b) Flow-induced voltage as a function of electrochemical bias voltage $\left(V_{\mathrm{B}}\right)$ for $0.01 \mathrm{M} \mathrm{KCl}, G_{0}=1.4 \mathrm{mS} / \mathrm{cm}$ (open circles) and $0.01 \mathrm{M} \mathrm{HCl}, G_{0}=2 \mathrm{mS} / \mathrm{cm}$ (filled circles). The solid line is a guide to the eye.

\subsection{Theoretical understanding}

There have been a number of attempts to understand the underlying physics behind the generation of electrical signals by the liquid flow over nanotubes. Král and Shapiro [9] had theoretically predicted the effect of electrical current generation in a metallic carbon nanotube immersed in a flowing liquid. However, their theory predicted a linear dependence of the flow-induced signals on the flow velocity. The other attempt invoked the idea of 'pulsating asymmetric ratchets' [10]. It suggested a transport mechanism where the pulsating asymmetric ratchets formed by the liquid flow over the nanotubes are responsible for the flow-induced current as well as the saturating response [1]. The features essential for the rectifying mechanism of the pulsating ratchet to operate are the spatial bias (asymmetry) obtained by the shear-induced deformation of the ion-plus-polar atmosphere at the liquidsolid interface and the temporal modulation is obtained from the liquid flow. The rectifying mechanism producing directed voltage based on the pulsating asymmetric ratchets qualitatively explains the data, both the direction of the flow-induced voltage with respect to the flow direction in the presence of different ionic species near the solid-liquid interface [6] and the saturating response [1]. However, an exact mapping would involve details regarding the dynamics of the ions in the presence of flow at the liquid-solid interface. An alternative attempt invoked the asymmetry in the force-force correlation function as seen by the charge carriers in the nanotubes [2]; this in a broad sense subsumed the asymmetric fluctuating ratchets [1] in a general way. The main idea behind this model is that the fluctuating Coloumbic field of the ions in the liquid offers a frictional grip on the charge carriers of the 

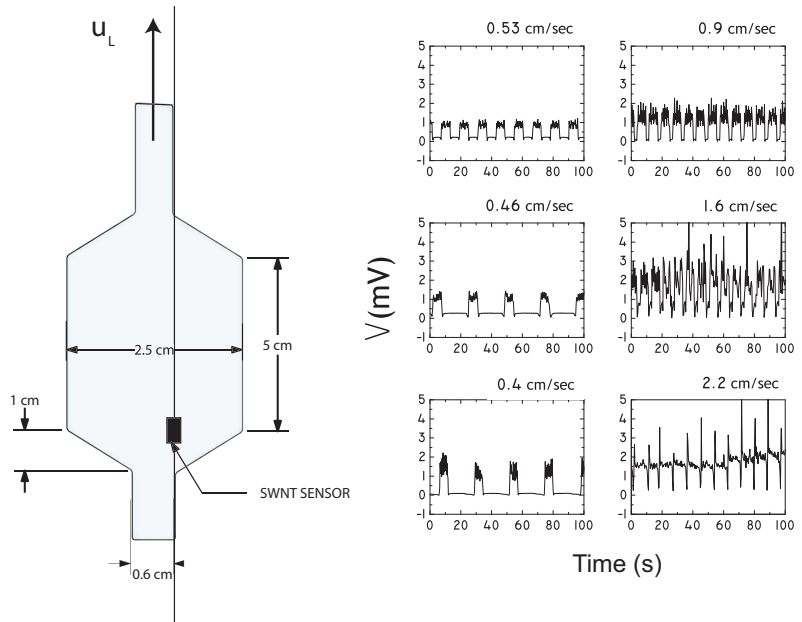

Time (s)

Figure 4. Left: The expansion geometry in which the sensor is kept. Right: The flow-induced voltage, generated as a function of the various average flow velocities.

nanotubes. Thus, as the charge carriers are advected by the fluid motion, a finite electrical current is generated. However, due to 'Doppler shift' in the force-force correlation function subtended by the ions in the liquid on the the charge carriers in the nanotubes, the aforementioned frictional grip reduces as a function of velocity, reproducing the sublinearity as seen in the experiments [2]. A similar result is obtained in Brownian dynamics simulation of a similar problem [11].

In the above analysis the variation of the velocity in the boundary layer at the surface of the nanotube has not been accounted for. We give a very simple argument here to discuss the effect of velocity variation in the boundary layer [12]. We find that the theoretical plot of flow-induced current to the flow velocity based on the asymmetry in the force-force correlation function as seen by the charge carriers in the nanotubes can be fitted [2] to the function, $I=I_{0} u_{\mathrm{L}} /\left(1+c u_{\mathrm{L}}\right)$. This equation can be generalized to include velocity variation by dividing the boundary layer formed at the nanotube-liquid interface into a number of layers, each moving with a constant velocity. We assume that the velocity of each layer increases linearly as we go away from the nanotube surface. Since the electrostatic interactions between the ions in the liquid and carriers in the nanotube vary inversely with the distance, it is reasonable to assume that the effectiveness of ions in a shell with velocity $u_{\mathrm{L}}$ to drag the carriers in the nanotube scale as $1 / u_{\mathrm{L}}$. Thus,

$$
I=I_{0} \int_{0}^{u_{\mathrm{L}}} \frac{u_{\mathrm{L}}^{\prime}}{1+c u_{\mathrm{L}}^{\prime}} \frac{\mathrm{d} u_{\mathrm{L}}^{\prime}}{u_{\mathrm{L}}^{\prime}}=I_{0} \log \left(1+c u_{\mathrm{L}}\right) .
$$

This simple idea interestingly reproduces the logarithmic dependence of the flowinduced signals on flow speeds. We do not want to overstretch the good matching of the data with eq. (2) because it is based on a very simplistic model. Here, we would also like to add that more than one mechanisms could very well be at work in these systems. In a recent publication, Persson et al [13] have invoked a combination 

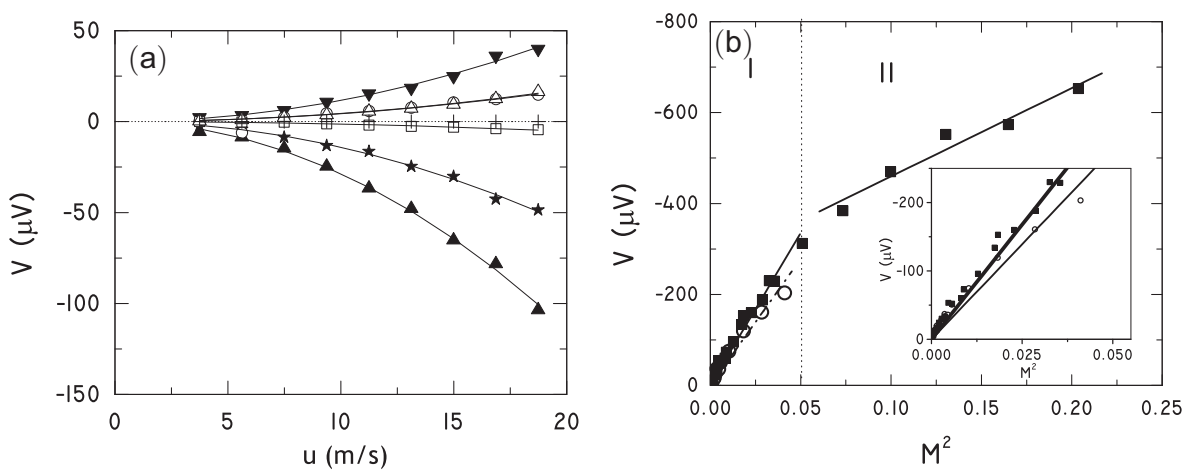

Figure 5. (a) Dependence of the signal $V$ on the flow velocity of nitrogen for (bottom to top) n-Si (filled up triangles), n-Ge (stars), graphite (open squares), Pt metal (plus), SWNT (open circles), MWNT (open up triangles) and $\mathrm{p}-\mathrm{Si}$ (filled down triangles). The solid lines are fit to $V=D u^{2}$, where $D$ is the fitting parameter [3]. (b) $V$ vs. $M^{2}$ for the flow of argon (filled squares) and nitrogen (open circles). The lines show fit to linear equation. The inset shows the expanded plot of regime I.

of frictional stick-slip and barrier-hopping to explain the observed phenomenon of flow-induced voltages in SWNT.

It has been suggested [14] that the flow-induced voltage can be explained simply in electrokinetic terms as the well-known streaming potential that develops along the flow of an electrolyte through a microporous insulator. However, the fact that the flow-induced signals have a strong sublinear, almost logarithmic variation with the flow velocities and strong dependence on the ionic strength rules out quite decisively the electrokinetic mechanism of voltage generation.

\section{Generation of electrical signals by flow of gases over solids}

Having seen that the flow of liquids over nanotubes generates electrical signals it was only natural to look for a similar effect by the flow of gas. We found [3] that gas flow also generates electrical signals which depended quadratically on the gas flow velocity. Further it was also found that the effect was not unique to nanotubes and occurred for a variety of solids such as single crystals of n-type Ge, n-Si, pSi, SWNT, multi-wall carbon nanotubes (MWNT), graphite and polycrystalline copper to establish the ubiquity of the gas flow-induced voltage generation. Figure 5 a shows the dependence of the voltage generated on the flow speed of nitrogen gas for p-Si, n-Si, n-Ge, SWNT, MWNT and graphite. It can be clearly seen that the voltage $V$ generated varies as $u^{2}$ over a wide range of $u$ (as does the current, not shown). Figure 5 shows the fit to $V=D u^{2}$ with the fit parameter $D$ [3]. It can be seen that the sign of the signal for p-type Si and SWNT is opposite to that for n-type Si, n-Ge, graphite and copper.

Figure 5b shows the generated voltage $V$ over a large range of values of $M^{2}$ for the flow of argon (solid squares) and nitrogen gases (open circles) over n-Ge where 


\section{A K Sood, S Ghosh and Anindya Das}

$M$ is the Mach number given by $M=u / c, c$ being the sound velocity $(=353 \mathrm{~m} / \mathrm{s}$ for nitrogen and $323 \mathrm{~m} / \mathrm{s}$ for argon at $300 \mathrm{~K}$ ). The use of $M^{2}$ is motivated by the theory to be presented later. A change in slope is clearly seen around $M^{2} \leq 0.05$. The inset in figure $5 \mathrm{~b}$ clearly shows that the slope $(\mathcal{A})$ for $M^{2} \leq 0.05$ (called regime I) is higher for argon as compared to that for nitrogen. The ratio of slopes $\mathcal{A}($ argon $) / \mathcal{A}($ nitrogen $)=1.2$. That there are actually two $M^{2}$-regimes is clear from our theoretical analysis of the mechanism behind the generation of electrical signal induced by the flow of gases over the solids and is discussed below.

\subsection{Theoretical understanding}

For adiabatic steady inviscid flow of a gas, Bernoulli's equation gives the pressure difference along a streamline in terms of Mach number $M$ as [15]

$$
\frac{P}{P_{0}}=\left[1-\frac{1}{2}(\gamma-1) M^{2}\right]^{\gamma /(\gamma-1)} \cong 1-\frac{\gamma}{2} M^{2} \quad\left(\text { for } \gamma M^{2} \ll 1\right),
$$

$\gamma \equiv C_{\mathrm{p}} / C_{\mathrm{v}}, ; C_{\mathrm{p}}\left(C_{\mathrm{v}}\right)$ being the specific heat at a constant pressure(volume). The values of $\gamma$ for argon and nitrogen are 1.667 and 1.404, respectively. In eq. (3), $P_{0}$ is the maximum pressure at a point on the streamline where velocity is zero. Such a point is the leading edge on the surface of the sample past which the gas is moving and is called the stagnation point. For the sample geometry the pressure difference between the two ends of the active sample exposed to the gas flow (i.e. without the electrodes) is hence $\left(P_{\mathrm{L}}-P_{\mathrm{R}}\right) / P_{0} \simeq \frac{\gamma}{2}\left(M_{\mathrm{R}}^{2}-M_{\mathrm{L}}^{2}\right)$. The subscripts $\mathrm{L}(\mathrm{R})$ denote the left(right) of the active sample when the gas flows from left to right. From the ideal gas law, the fractional temperature difference $\Delta T / T$ is related to the pressure difference $\Delta P / P$ and the density difference $\Delta \rho / \rho$ as $\Delta T / T=\Delta P / P-\Delta \rho / \rho$. When $M \ll 1$, called regime I, the change in density of the gas is negligible, i.e. the fluid is essentially incompressible and hence $\Delta T / T=\Delta P / P$. Beyond a certain value of $M(\sim 0.2)$, called regime II, the density changes of the gas should be taken into account which gives [15] $\Delta T / T_{0} \simeq \frac{1}{2}(\gamma-1)\left(M_{\mathrm{R}}^{2}-M_{\mathrm{L}}^{2}\right)$. The gas flowing past the sample kept at an angle $\alpha$ with respect to the horizontal axis corresponds to an accelerating flow and hence $M_{\mathrm{R}}>M_{\mathrm{L}}$. The temperature difference along the streamline in the gas flow will induce a temperature difference in the solid along the flow direction. The temperature difference, in turn, will result in a voltage difference $V$, defined as $V_{\mathrm{L}}-V_{\mathrm{R}}$, due to the Seebeck effect. We can write

$$
V= \begin{cases}\frac{k}{2} T_{0} \gamma S\left(M_{\mathrm{R}}^{2}-M_{\mathrm{L}}^{2}\right) \propto \frac{k T_{0}}{2} \gamma S M^{2} & \text { Regime I }(M<0.2) \\ \frac{k}{2} T_{0}(\gamma-1) S\left(M_{\mathrm{R}}^{2}-M_{\mathrm{L}}^{2}\right) \propto \frac{k T_{0}}{2}(\gamma-1) S M^{2} & \text { Regime II }(M>0.2),\end{cases}
$$

where $S$ is the Seebeck coefficient of the solid, positive for p-type (majority carries as holes) and negative for n-type materials. The factor $k$ depends on the specific interactions between the gas and the solid surface as well as on the boundary conditions of the temperature difference between the gas and the solid.

We have seen that the various predictions of our model such as the dependence of the signal on the square of the Mach number $(M)$, the dependence of the strength 
and magnitude of the signal on the Seebeck coefficient of the material, the dependence of the signal on the exposed length of the sample and on the angle of inclination $(\alpha)$ with respect to the flow direction and the ratio of slopes in the regions I and II of figure 5b agree well with the theory presented above [3].

Our experiments clearly show that a sensor to measure the flow velocity of the gases can be made based on the generated electrical signal. It is an active sensor which gives direct electrical response to the gas flow. This should be compared with the widely used gas flow sensor based on thermal anemometry, wherein, the fluid velocity is sensed by measuring changes in heat transfer from a small, electricallyheated sensor (wire or thin film) exposed to the fluid. Even though we have shown results only for a few solids we emphasize that the effect is not restricted to these materials alone. The guiding principle for the choice of the solid is its high Seebeck coefficient, which will suggest other solids like selenium, tellurium, $\mathrm{Bi}_{2} \mathrm{Te}_{3}, \mathrm{GaAs}$, oxides and electrically conducting polymers.

\section{Acknowledgements}

We thank Prof. N Kumar, Prof. S Ramaswamy and Prof. C N R Rao for their active and enjoyable collaboration. AKS thanks the Department of Science and Technology (Government of India) for financial support.

\section{References}

[1] S Ghosh, A K Sood and N Kumar, Science 299, 1042 (2003)

[2] S Ghosh, A K Sood, S Ramaswamy and N Kumar, Phys. Rev. B270, 205423 (2004)

[3] A K Sood and S Ghosh, Phys. Rev. Lett. 93, 86601 (2004) and references therein

[4] P V Teredesai, A K Sood, D V S Muthu, R Sen, A Govindraj and C N R Rao, Chem. Phys. Lett. 319, 296 (2000)

[5] The sensor used for obtaining the data in figure 2 is different from that used to obtain the data shown in figure 1 and hence the absolute values of the signals shown in figures (1) and (2) are not to be compared

[6] A K Sood, Radiat. Phys. Chem. 70, 647 (2004)

[7] H K Moffatt, J. Fluid. Mech. 18, 1 (1964)

[8] A K Sood, A Das and S Ghosh (Patent applied)

[9] P Král and M Shapiro, Phys. Rev. Lett. 86, 131 (2001)

[10] P Reimann, Phys. Rep. 290, 149 (1997)

[11] M Das, S Ramaswamy, A K Sood and G Ananthakrishna, arXiv: cond-mat 10505017

[12] A K Sood, S Ghosh and N Kumar (unpublished)

[13] B N J Persson, U Tartaglino, E Tosatti and H Ueba, Phys. Rev. B69, 235410 (2004)

[14] A E Cohen (comment), S Ghosh, A K Sood and N Kumar (reply), Science 300, 1235 (2003)

[15] L D Landau and E M Lifshitz, Fluid mechanics, 2nd Edition (Butterworth Heinemann, 1998) p. 319

[16] L D Landau and E M Lifshitz, Physical kinetics, 2nd Edition (Butterworth Heinemann, 1998) p. 51 